\title{
A NOTE ON THE THREE-PORTFOLIOS MATCHING PROBLEM
}

\author{
FABIO TROJANI, PAOLO VANINI, AND LUIGI VIGNOLA
}

\begin{abstract}
A typical problem arising in the financial planning for private investors consists in the fact that the initial investor's portfolio, the one determined by the consulting process of the financial institution and the universe of instruments made available to the investor have to be matched/optimized when determining the relevant portfolio choice. We call this problem the three-portfolios matching problem. Clearly, the resulting portfolio selection should be as close as possible to the optimal asset allocation determined by the consulting process of the financial institution. However, the transition from the investor's initial portfolio to the final one is complicated by the presence of transaction costs and some further more specific constraints. Indeed, usually the portfolios under consideration are structured at different aggregation levels, making portfolios comparison and matching more difficult. Further, several investment restrictions have to be satisfied by the final portfolio choice. Finally, the arising portfolio selection process should be sufficiently transparent in order to incorporate the subjective investor's trade-off between the objectives 'optimal portfolio matching' and 'minimal portfolio transition costs'. In this paper, we solve the three-portfolios matching problem analytically for a simplified setting that illustrates the main features of the arising solutions and numerically for the more general situation.
\end{abstract}

Keywords: Transaction Costs, Portfolio Matching, Portfolio Selection

JEL-Classification: G2, G11, G14, G21

Date: This Version: July 25, 2001.

Fabio Trojani, Insitute of Finance, University of Southern Switzerland, Via G. Buffi 13, CH-6900 Lugano, e-mail: fabio.trojani@lu.unisi.ch. Fabio Trojani gratefully acknowledges the financial support of the Swiss National Science Foundation (grant 1214-056679).

Paolo Vanini (corresponding author), Zürcher Kantonalbank, Corporate Risk Control, Neue Hard 9, CH-8000 Zurich, e-mail: paolo.vanini@zkb.ch.

Luigi Vignola, Zürcher Kantonalbank, Trading: Models and Methods, Uraniastrasse 31+35, CH8001 Zurich, e-mail: luigi.vignolaezkb.ch. 


\section{INTRODUCTION}

In the financial planning for private investors the consulting company (i.e. a bank) often faces the problem that the initial investor's portfolio, the one determined by the consulting process of the bank and the universe of instruments made available to the investor have to be matched/optimized when determining the relevant portfolio choice. We call this problem the three-portfolios matching problem (TPMP).

Investors typically have some initial portfolio consisting of securities and cash. In the bank's consulting process a corresponding optimal asset allocation adapted to the subjective investor's risk/return profile is then determined. To define the optimal asset allocation the bank typically defines a set of so-called asset classes ${ }^{1}$, which are simply specific sets of securities having at least one common characteristic ${ }^{2}$ (cf. also Section 5, Table 1, for some more concrete examples of asset classes). In order to adapt the initial portfolio to the target, the bank offers to the investor a specific set of assets for trading, the bank's investment universe. These instruments are typically funds ${ }^{3}$.

In the absence of transaction costs and restrictions on the available investment universe the bank can easily solve the TPMP by liquidating the initial portfolio, collecting the proceedings, and finally simply investing in a portfolio that exactly matches the given optimal asset allocation. However, if transaction costs are considered or if the investment universe supplied by the bank is constrained, this strategy is generally neither optimal nor feasible. For instance, investor's initial portfolio might already largely satisfy the porfolio structure implied by the optimal asset allocation ${ }^{4}$, or exact matching might not even be possible with the available investment vehicles.

As a consequence, a systematic approach taking transaction costs and investment constraints into account is needed. Basically, the problem at hand is to determine a (static) trading strategy such that

(1) The final portfolio is as 'close' as possible to the target asset allocation (this is the matching objective),

(2) The transaction costs caused by the portfolio transition are minimal (this is the cost objective),

\footnotetext{
${ }^{1}$ The cardinality of this set is typically lower than that of the set of universally available investment vehicles. However, if the asset classes are well-diversified, a well-diversified optimal asset allocation follows.

${ }^{2}$ After having identified the asset classes, the typical procedure is to choose one representative security or index (for example the Swiss Market Index (SMI)) and to use its characteristics as the representative characteristics of the asset class itself. For bonds, asset classes are characterized by the currency and by the time to maturity. Another characteristic could be the rating (if there is any), but we assume that for private investors only top-rated instruments are considered as admissible investment opportunities.

${ }^{3}$ The cardinality of the set of funds offered to the investor typically depends on her initial wealth.

${ }^{4}$ This is similar to the problem of sorting a list of elements. If the list is already sorted, any sorting effort will be completely inefficient. That's why many sorting algorithms do indeed reshuffle the complete list in a random way before actually starting to sort.
} 
(3) The imposed investment restrictions are considered.

In order to solve this problem, portfolios are aggregated on the asset class level. Indeed, typically a large part of the investor's initial portfolio can be aggregated in some set of predefined asset classes. The remaining part is then simply mapped to a residual class. Besides 'pure' asset classes such as 'stocks' or 'bonds', investment funds consisting of a mix of other asset classes (and hence of the respective characteristics) are also included in portfolio management. In the sequel we assume that a fund can always be represented as a linear combination of asset classes.

The three portfolios that determine the TPMP are most of the times organized in a very different way (usually using different aggregation levels) making a direct portfolio comparison or matching often unnatural. However, using the notion of asset classes they can be naturally related one to each other. For instance, suppose that the investor initially owns twenty different securities. Further, assume that the bank has identified ten different asset classes and has setted an optimal asset allocation by defining percentages of initial wealth that have to be invested in twenty funds supplied by the bank (cf. for instance Table 5 in the Appendix). From an abstract point of view, the TPMP then consists of finding a ten dimensional vector (the trading vector for the initial portfolio) and twenty ten-dimensional vectors (the fund's trading vectors) such that, first, some distance measure between the final investor's portfolio and the target one is minimized and, second, the resulting transaction costs are taken into account. In this respect, a few important questions have to be addressed when defining a solution procedure for this problem:

(1) What is a 'good' distance measure between the initial portfolio and the target?

(2) How can transaction costs be taken into account?

(3) How does the trade-off between the selling of existing assets and the buying of new funds look like?

Notice that in offering a practical solution proposal to the TPMP we will not assume (as is implicitly done in many research papers) that the objective function on which the bank's optimal asset allocation policy has been funded is known to the department selecting the matching portfolio of private investors. Indeed, in practice the departments determining the bank's optimal asset allocation policy and those selecting the portfolios of private investors are most of the times completely disjoint entities. Moreover, the objective function behind the choosen optimal asset allocation is practically never made really explicit to the portfolio managers. Therefore, approaches that try to optimize some indicator related to a concept of efficiency loss like for instance the maximum correlation between two relevant portfolios (Kandel and Stambauch $(1987,1989)$ and Schanken (1987)) or a maximal variance (expected return) difference for a given expected return (variance) level (Kandel et al. (1995) and Wang (1998)) are not really feasible or reasonable in the present setting.

In this paper we address questions $1 .-3$. by presenting a possible solution way to the TPMP, which is feasible (and reasonable) for practical purposes. We first consider analytical expressions for a simplified model which illustrates some of the main features of the arising solutions. For this case, we describe explicitly the 
trade-off between the selling of existing assets and the buying of new funds, both in the presence and in the absence of transaction costs. In a second step we address the more general case and present numerical computations for an example from the practice.

\section{THE MODEL}

We assume that there are $N$ disjoint asset classes such that every (pure) security can be attributed exactly to one asset class. All initial portfolio positions not belonging to one of these asset classes are collected in a residual asset class.

The exogenous target asset allocation determined in the consulting process of the bank is represented by a vector of weights ${ }^{5}$

$$
\lambda^{*}=\left(\lambda_{1}^{*}, \ldots, \lambda_{N}^{*}\right)^{\prime} \text { with } \sum_{j=1}^{N} \lambda_{j}^{*}=1 .
$$

The optimal allocation of initial wealth $W_{0}$ to the asset class $j$ is then simply $\lambda_{j}^{*} W_{0}$. Notice that initial Wealth $W_{0}$ is typically expressed with respect to a given reference currency ${ }^{6}$.

The investor's initial portfolio is represented by a vector $\lambda=\left(\lambda_{1}, \ldots, \lambda_{N}\right)^{\prime}$ of weights assigned to the asset classes specified in the bank's optimal asset allocation. With this notation, the amount of residual wealth $R$ invested in the residual asset class is

$$
R=W_{0}\left(1-\sum_{j=1}^{N} \lambda_{j}\right)=W_{0} \sum_{j=1}^{N}\left(\lambda_{j}^{*}-\lambda_{j}\right) .
$$

The potential selling or buying positions of the investor (i.e. the marginal increase or decrease of portfolio holdings) are represented by a vector of weights $b=\left(b_{1}, \ldots, b_{N}\right)^{\prime}$. The $b_{j}$ 's are decision variables in our TPMP and the change $\Delta P$ in the investor's portfolio value implied by a policy $b$ is

$$
\Delta P=W_{0} \sum_{j=1}^{N} b_{j} .
$$

The $k^{\text {th }}$ fund, $k=1 . . K$, is represented by a vector of weights $\mu^{(k)}=\left(\mu_{1}^{(k)}, \ldots, \mu_{N}^{(k)}\right)^{\prime}$, such that

$$
\mu_{j}^{(k)} \in[0,1] \text { and } \sum_{j=1}^{N} \mu_{j}^{(k)} \leq 1 .
$$

Hence, not all weights of the fund might be attributed to the asset classes defined in the bank's optimal asset allocation. Similarly to the above definition of $\Delta P$, the

\footnotetext{
${ }^{5}$ The optimal asset allocation could be determined for example using a static Markowitz (1956)type or a dynamic Merton (1969, 1971)-type model. However, in this paper we do not explicitly model the process of determining optimal asset allocations.

${ }^{6}$ When investments in different currencies are admitted we assume that they are expressed in a single numéraire, as for instance the home currency of our investor.
} 
change $\Delta F$ in the value of the aggregate portfolio of funds is

$$
\Delta F:=W_{0} \sum_{k=1}^{K} a_{k},
$$

where $a=\left(a_{1}, . ., a_{k}\right)^{\prime} \in[-1,1]^{K}$ is a vector of marginal buying/selling positions $a_{j}$ in the $j$-th fund. The $a_{j}$ 's also are decision variables in our TPMP problem.

For both the transactions $b$ in the initial portfolio and the transactions $a$ in the funds costs arise, which we assume to be proportional to the absolute transaction amount $^{7}$, that is

$$
T^{(P)}(b)=\sum_{j=1}^{N}\left|b_{j} W_{0}\right| c_{j}^{(P)} \quad \text { with } c_{j}^{(P)} \geq 0
$$

and

$$
T^{(F)}(a)=\sum_{k=1}^{K}\left|a_{k} W_{0}\right| c_{k}^{(F)} \quad \text { with } c_{k}^{(F)} \geq 0 .
$$

Hence, total transaction costs are given by

$$
T(a, b)=T^{(P)}(b)+T^{(F)}(a) .
$$

In order to obtain a functional form for (2.1) and (2.2) that is more suitable for optimization, we further linearize the total transaction costs function to get

$$
\begin{aligned}
\hat{T}\left(\alpha^{ \pm}, \beta^{ \pm}\right) & =\hat{T}^{(P)}\left(\beta^{ \pm}\right)+\hat{T}^{(F)}\left(\alpha^{ \pm}\right) \\
& =\sum_{j=1}^{N}\left(\beta_{j}^{+}+\beta_{j}^{-}\right) W_{0} c_{j}^{(P)}+\sum_{k=1}^{K}\left(\alpha_{k}^{+}+\alpha_{k}^{-}\right) W_{0} c_{k}^{(P)}
\end{aligned}
$$

with the additional constraints

$$
\begin{array}{ll}
b_{j}=\beta_{j}^{+}+\beta_{j}^{-}, & j=1 . . N \\
a_{k}=\alpha_{k}^{+}+\alpha_{k}^{-} & k=1 . . K
\end{array}
$$

and

$$
\alpha_{k}^{+}, \alpha_{k}^{-}, \beta_{j}^{+}, \beta_{j}^{-} \geq 0
$$

The major issue in determining the final investment proposal basen on the available funds is the definition of a reasonable criterion by which the bank's optimal asset allocation and the allocation to the various funds can be compared. At first sight, a simple criterion such as the squared euclidean distance

$$
\delta_{k}:=\left\|\lambda^{*}-\mu^{(k)}\right\|^{2}:=\left(\lambda^{*}-\mu^{(k)}\right)^{\prime}\left(\lambda^{*}-\mu^{(k)}\right)
$$

between the optimal allocation $\lambda^{*}$ and a fund allocation $\mu^{(k)}$ might be adopted. Such a criterion has however some weaknesses. Indeed, if the TPMP would simply read

\footnotetext{
${ }^{7}$ Further non-linear cost functions can be in principle considered, if one is ready to take into account a higher complexity of the optimization procedure described in Section 3 below.
} 
"Find one single fund that minimizes the euclidean distance between $\lambda^{*}$ and (some) $\mu^{(k)}$,

we would be - at least in principle - easily done. However, with this criterion we are unable to definitely handle situations where distances between asset allocations are equal but arise within different asset classes. For instance, for an optimal asset allocation $\lambda^{*}=(0.7,0.3)^{\prime}$ into a bond and an equity index, say, two funds with $\mu^{(1)}=(0.6,0.4)^{\prime}$ and $\mu^{(2)}=(0.8,0.2)^{\prime}$ would imply an identic distance $\delta_{j}=$ 0.02 . Hence, from a pure euclidean distance perspective the two funds have to be considered both as optimal, respectively equally feasible. However, if equity is a riskier asset than bonds, investing in the first fund would possibly neglect the customers guidance relatively to its risk exposure.

Clearly, different solution ways to this situation can be pursued. For instance, one could limit the exposure to the riskiest asset and thereby prefer to invest in the first fund only. A further possibility is to split wealth equally between the two funds. In some situations however, also this proposal may be of no use. Consider for instance a situation with three asset classes and allocative constraints that do not permit short-selling of funds. In this case, a target asset allocation might not even be reached at all. For example let $\lambda^{*}=(0.1,0.5,0.4)^{\prime}$ and consider an universe of funds represented by the matrix

$$
\mathbf{M}=\left(\mu^{(1)}, \mu^{(2)}, \mu^{(3)}\right)=\left(\begin{array}{lll}
0.2 & 0.5 & 0.2 \\
0.2 & 0.5 & 0.4 \\
0.6 & 0.0 & 0.4
\end{array}\right) \text {. }
$$

Since the only solution $x$ to the linear system $\mathbf{M} x=\lambda^{*}$ is $x=(-2 / 3,-1 / 3,2)^{\prime}$ and involves the short-selling of the first two funds, exact matching of the target while satisfying the given constraint is in this case impossible.

The shortcomings of a pure euclidean distance as the objective that has to be optimized cannot be completely eliminated. They can however be weakened by introducting additional requirements on the final portfolio choice that have to be satisfied (cf. again the above simple examples). Three desirable properties of a portfolio selection criterion for private investors are the following.

Property P.1 (No excess risk exposure). In the final portfolio choice the relative propensity to invest in the riskiest asset categories, as defined by the bank's optimal asset allocation (typically the sum over all weights in the equity asset classes) cannot be exceeded.

Property P.2 (Accounting for the initial portfolio). The initial asset allocation $\lambda$ has to be taken into account.

Property P.3 (Parsimony). If $\lambda^{*}$ can be achieved through different portfolios of funds, the one with the smallest number of different funds has to be preferred.

While P.1 is economically motivated, P.2 and P.3 are purely justified by the presence of transaction costs. The next sections present an optimization algorithm that indeed solves the TPMP consistently with P.1, P.2 and P.3. 


\section{OPTIMIZATION PROBLEM}

In this section we start with a simplified formulation of a TPMP that neglects P.1 and P.3 above. The optimization problem relevant for this section is

$$
Q(a, b)=\min _{a, b} \frac{1}{2}\left\|\lambda^{*}-\left(\lambda+b+\sum_{k=1}^{K} a_{k} \mu^{(k)}\right)\right\|^{2}
$$

subject to

$$
\begin{gathered}
w=W_{0} \sum_{j=1}^{N}\left(b_{j}-\left[\sum_{k=1}^{K} a_{k} \mu^{(k)}\right]_{j}\right)-\hat{T}\left(\alpha^{ \pm}, \beta^{ \pm}\right) \\
=\Delta P-\Delta F-\hat{T}\left(\alpha^{ \pm}, \beta^{ \pm}\right) \geq 0, \\
b_{j}=\beta_{j}^{+}+\beta_{j}^{-}, \quad j=1 . . N \\
a_{k}=\alpha_{k}^{+}+\alpha_{k}^{-} \quad k=1 . . K
\end{gathered}
$$

and

$$
\alpha_{k}^{+}, \alpha_{k}^{-}, \beta_{j}^{+}, \beta_{j}^{-} \geq 0
$$

where $\hat{T}\left(\alpha^{ \pm}, \beta^{ \pm}\right)$is defined in (2.3), $w$ denotes the (additional) amount of money that needs to be invested, and (3.2) is the budget constraint. Specifically, (3.2) states that the sum of the transaction costs from buying/selling positions in the initial portfolio and in the funds, plus the netto amounts invested in the respective instruments, has to be equal to the amount of money $w$ at disposition of the investor. If $w \equiv 0$, all new transactions have to be financed through the selling of existing assets.

The interpretation of (3.1) deserves some (small) caution. For instance, in the absence of transaction costs and when the residual class is empty one might be tempted to conclude that the objective $Q(a, b)$ can be made arbitrary small. However, as the examples of the last section show, this is not generally true especially when investment constraints have to be satisfied.

Further, notice that (3.1) is a quadratic optimization problem, which is - at least formally - similar to the portfolio optimization problem in a Markowitz (1956)type model. Therefore, in the same vain as one can determine a mean-variance efficient frontier in that model, it is possible to define a cost-optimal matching efficient frontier for the TPMP. In mean-variance theory the relevant market parameters are the mean and the covariance matrix of asset returns. In our problem the relevant parameter is the difference vector

$$
\lambda^{*}-\left(\lambda+b+\sum_{k=1}^{K} a_{k} \mu^{(k)}\right)
$$

that defines the allocation differences between an investor's final portfolio choice and the target asset allocation of the bank. 


\section{Analytical Solution}

In this section, we solve problem (3.1)-(3.4) analytically and analyze some properties of its solution. From convex analysis we know that the inequality constraints in (3.2) lead to the slackness and the positivity conditions for the corresponding Kuhn-Tucker multipliers. Each of these conditions implies that a small number of asset classes has to be considered in order to derive analytical solutions. Fortunately, the impact of these restrictions on the unrestricted problem is known. Indeed, if none of the restrictions binds, the solution of the restricted problem is the one of the unrestricted problem. If one or more restrictions bind, the global shape of the unrestricted model is changed as follows. Suppose, that in a (transactions) cost-matching diagram, the global locus of cost-matching efficient portfolios are hyperbolae for all value of the parameters. Then, in the presence of restrictions a single hyperbola is replaced by a union of different hyperbolae, that are each defined on a subset of the parameter space.

Setting for simplicity $W_{0} \equiv 1$ we can rewrite problem (3.1) in matrix form, using the notation $\mathbf{I}$ for the $N \times N$ identity matrix and the notation

$$
\sum_{k=1}^{K} a_{k} \mu^{(k)}=\mathbf{M} a
$$

for some suitable $N \times K$ matrix $\mathbf{M}$. The next result characterizes the solution of the TPMP under the present simplifying assumptions.

Proposition 1. Consider problem (3.1) with $w=0$ and suppose that

$$
\mathbf{U}:=\left(\mathbf{M}^{\prime} \mathbf{M}\right)^{-1}
$$

exists. The solution to problem (3.1) is characterized by the relation

$$
a^{*}=\mathbf{U M}^{\prime}\left(\Lambda-b^{*}\right)+\frac{\gamma}{2} \mathbf{U}\left(c^{(F)}+\mathbf{M}^{\prime} c^{(P)}\right) ; \gamma \geq 0,
$$

between the optimal transactions in assets already own by the investor and those in the funds available for investment.

Proof. First order conditions imply

$$
\begin{aligned}
& 0 \equiv \frac{\partial Q}{\partial a}=\mathbf{M}^{\prime}[\Lambda-b-\mathbf{M} a]+\gamma\left(c^{(F)}+\mathbf{M}^{\prime} \mathbf{1}_{N}\right) \\
& 0 \equiv \frac{\partial Q}{\partial b}=-[\Lambda-b-\mathbf{M} a]+\gamma\left(c^{(P)}+\mathbf{1}_{N}\right)
\end{aligned}
$$

and hence

$$
0=2 \mathbf{M}^{\prime}[\Lambda-b-\mathbf{M} a]+\gamma\left(c^{(F)}+\mathbf{M}^{\prime} \mathbf{1}_{N}\right)-\gamma \mathbf{M}^{\prime}\left(c^{(P)}+\mathbf{1}_{N}\right) .
$$

Solving for $a$ and taking into account the inequality restrictions yields the desired result.

From (4.1) we can immediately infer a linear optimal trade-off between investment in funds and/or liquidation of existing assets. Indeed, we immediately have

$$
\frac{\partial a^{*}}{\partial b^{*}}=-\mathbf{M U}^{\prime}
$$


showing, as expected, that the optimal trade-off is closely related to the market characteristics of the given funds (which are synthesized by the matrix $\mathbf{M}$ ).

In order to get a further feeling for the behaviour of the trade-off implied by (4.1) set $c^{(P)}=\mathbf{0}_{N}$ and $c^{(F)}=\mathbf{0}_{K}$. In this case

$$
a^{*}=\mathbf{U M}^{\prime}\left(\Lambda-b^{*}\right) \text {, }
$$

that is, any difference left from the purchase or selling of assets in the intial investor's portfolio has to be compensated by a (properly scaled) investment in the funds.

To further incorporate P.1 and P.3 in problem (3.1) it is now sufficient to impose the additional constraint

$$
\hat{T}\left(\alpha^{ \pm}, \beta^{ \pm}\right) \leq T
$$

which sets an upper bound on the total transaction costs admissible for an investor. The arising problem can be then solved along the same lines as the simplified problem above, simply by imposing the additional restriction on the trade-off between $a$ and $b$.

\section{NUMERICAL EXAMPLE}

To illustrate the model further we consider a numerical example where we distinguish 15 asset classes. The asset classes are defined by a matrix presented in Table 1. We can interpret Table 1 as a representation of three 'super'-asset classes, Equity,

\begin{tabular}{|rrrrrrr|r|}
\hline & CHF & USD & EUR & JPY & GBP & Other & Total \\
\hline Equity & $40.0 \%$ & $15.0 \%$ & $5.0 \%$ & $1.0 \%$ & $0.0 \%$ & $0.0 \%$ & $61.0 \%$ \\
Bonds & $0.0 \%$ & $10.0 \%$ & $15.0 \%$ & $0.0 \%$ & $0.0 \%$ & $0.0 \%$ & $25.0 \%$ \\
Cash & $10.0 \%$ & $2.0 \%$ & $0.0 \%$ & $2.0 \%$ & $0.0 \%$ & $0.0 \%$ & $14.0 \%$ \\
Other & $0.0 \%$ & $0.0 \%$ & $0.0 \%$ & $0.0 \%$ & $0.0 \%$ & $0.0 \%$ & $0.0 \%$ \\
\hline \hline Total & $50.0 \%$ & $27.0 \%$ & $20.0 \%$ & $3.0 \%$ & $0.0 \%$ & $0.0 \%$ & $100.0 \%$ \\
\hline
\end{tabular}

TABLE 1. The optimal asset allocation of an investment consulting process. All numbers are in percentage of invested wealth and are expressed in the 'home currency' of the investor.

Bonds and Cash across five different currencies, with the understanding that some classes are purely equity in nature while other classes contain bonds with different maturities. In this example, we also have a residual asset class called 'Other' for each currency. The investment universe offered to our investor consists of 26 funds denoted by $A, B, \ldots, Z$, whose detailed composition in single asset classes is presented in Table 5 of the Appendix. We further assume that through some more or less sophisticated process the investor has determined an optimal asset allocation which is given in Table 1.

In order to make our example more realistic, we consider the following additional investment restrictions:

(i) The percentage of wealth that has to be invested is $100 \%$. Note that we consider the holding of cash as an investment in 'liquidity'. 
(ii) The relative weight of each single fund cannot exceed $60 \%$.

(iii) Short-selling of any fund is excluded.

(iv) The percentage of wealth invested in risky assets, i.e. in equity, cannot exceed $75 \%$.

(v) To show the flexibility of the approach we further assume that any optimal investment less than $5 \%$ of total wealth has to be set to zero and that investment is restricted to a maximum of five different funds.

For simplicity, we exclude in this example transaction costs even if they can be easily incorporated by the algorithm.

\begin{tabular}{|cr|}
\hline Name of fund & $\begin{array}{r}\text { Amount of initial } \\
\text { wealth to be invested }\end{array}$ \\
\hline Fund J & $58.475 \%$ \\
Fund N & $7.623 \%$ \\
Fund O & $9.629 \%$ \\
Fund T & $16.750 \%$ \\
Fund V & $7.523 \%$ \\
\hline \hline Total & $100.000 \%$ \\
\hline
\end{tabular}

TABLE 2. Investment proposal on the basis of five funds and the restrictions explained in the text.

Starting from the optimal asset allocation in Table 1 and running through the optimization we obtain the transactions (i.e. purchases of funds) required for best matching. They are displayed in Table 2 . The resulting absolute differences with respect to the target optimal asset allocation are given in Table 3 and shown in Figure 1 below.

\begin{tabular}{|rrrrrrr|r|}
\hline Equity & $0.14 \%$ & $0.12 \%$ & $0.85 \%$ & $3.68 \%$ & $0.00 \%$ & $0.00 \%$ & $4.79 \%$ \\
Bonds & $0.00 \%$ & $0.04 \%$ & $0.11 \%$ & $0.00 \%$ & $0.00 \%$ & $0.00 \%$ & $0.15 \%$ \\
Cash & $4.15 \%$ & $0.92 \%$ & $0.00 \%$ & $2.00 \%$ & $0.00 \%$ & $0.00 \%$ & $7.08 \%$ \\
Other & $0.00 \%$ & $0.00 \%$ & $0.00 \%$ & $0.00 \%$ & $0.00 \%$ & $0.58 \%$ & $0.58 \%$ \\
\hline \hline Total & $4.3 \%$ & $1.1 \%$ & $1.0 \%$ & $5.7 \%$ & $0.0 \%$ & $0.6 \%$ & $0.0 \%$ \\
\hline
\end{tabular}

TABLE 3. Absolute differences between the optimal asset allocation and the asset allocation achieved by investing in the funds as proposed in Table 2 .

If we weaken some investment restrictions by allowing for investment in up to 10 different funds, the results of the optimization change only slightly as shown in Table 4.

\section{CONCLUSION}

In this paper we solved a three-portfolios optimal matching problem for private investors. The model leads to some interesting insights for asset management and 


\begin{tabular}{|cr|}
\hline Name of fund & $\begin{array}{r}\text { Amount of initial } \\
\text { wealth to be invested }\end{array}$ \\
\hline Fund B & $0.200 \%$ \\
Fund J & $58.24 \%$ \\
Fund N & $7.592 \%$ \\
Fund O & $9.590 \%$ \\
Fund R & $0.100 \%$ \\
Fund S & $0.100 \%$ \\
Fund T & $16.683 \%$ \\
Fund V & $7.493 \%$ \\
\hline \hline Total & $100.000 \%$ \\
\hline
\end{tabular}

TABLE 4. Investment proposal on the basis of ten funds and the restrictions explained in the text.

products designing teams in the banks. We demonstrated that a not fully overlapping selection of the asset classes used in determining the optimal asset allocation and those behind the instruments (funds) available to the clients can lead to some very unpleasant consequences for the clients. Indeed, on the one side they are a priori constrained in determining a final portfolio that has to be close to the target investment proposal of the bank. On the other hand, they can realize serious difficulties in achieving the target using the investment vehicles supplied by the bank. This is a quite paradoxical situation, since both the investment proposal and the impossibility to reach the target are determined by the same institution. Therefore,

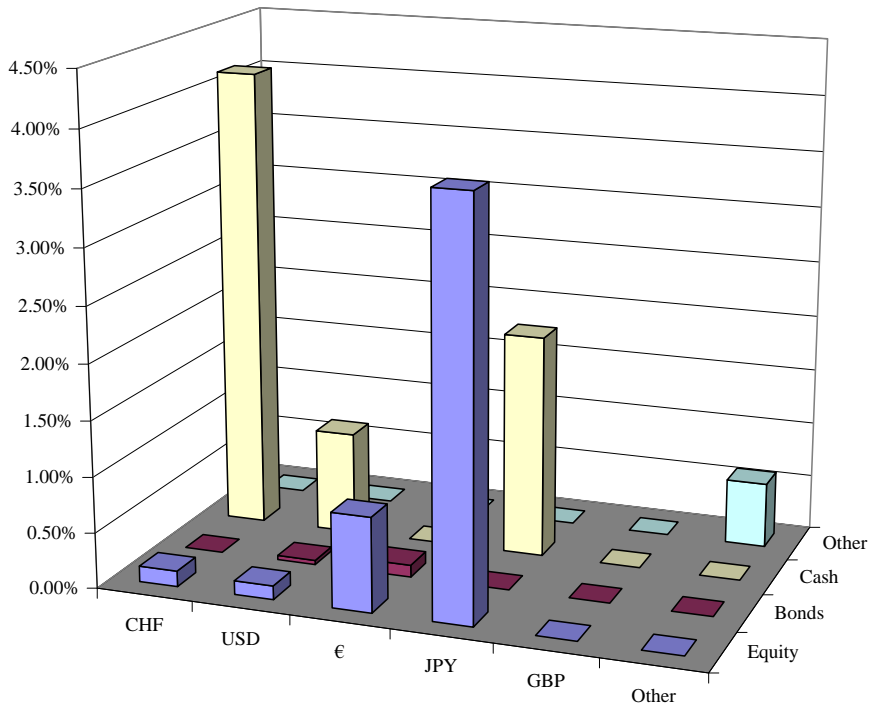

FIGURE 1. Absolute differences between the obtained asset allocation and the optimal asset allocation in Table 1, when investment is undertaken following the proposal of Table 2 . 
a serious financial planning has to be careful about the joint selection of the relevant asset universe and the definition of the optimal asset allocation for the clients.

We proposed an algorithm to solve TPMP that take into account possible inconsistencies between optimal asset allocations and universe of available intruments in a reasonable way. The solution of the model led to a mean transaction costs-optimal portfolio matching efficient frontier which can be conveniently used in the consulting process of private investors. This curve makes the trade-off between optimal portfolio matching and the induced transaction costs simple to understand and to communicate. 


\section{REFERENCES}

Kandel, S., R. Mc. Culloch, and R. Stambaugh (1995), Bayesian Inference and Portfolio Efficiency, Review of Financial Studies 8, 1-53.

KANDEL, S., and R. STAMBAugh (1987), On Correlation and Inferences about Mean-Variance Efficieny, Journal of Financial Economics 18, 61-90.

KAndel, S., and R. Stambaugh (1989), A Mean-Variance framework for Tests of Asset Pricing Models, Review of Financial Studies 2, 125-156.

Markowitz, H.M. (1956), The Optimization of a Quadratic Function Subject to Linear Constraints, Naval Research Logistics Quarterly 3, 111-133

Merton, R.C. (1969), Lifetime Portfolio Selection under Uncertainty: The Continuous-Time Case, Review of Economics and Statistics 51, 247-256

Merton, R.C. (1971), Optimum Consumption and Portfolio Rules in a ContinuousTime Model, Journal of Economic Theory 3, 373-413.

SCHANKEN, J. (1987), Multi-Variate Proxies and Asset pricing Relations: Living with Roll's Critique, Journal of Financial Economics 18, 91-110.

WANG, Z. (1998), Efficiency Loss and Constraints on Portfolio Holdings, Journal of Financial Economics 48, 359-375. 


\section{APPENDIX}

\begin{tabular}{|c|c|c|c|c|c|c|c|c|c|c|}
\hline & & Fund $A$ & Fund B & Fund C & Fund D & Fund E & Fund F & Fund G & Fund $\mathrm{H}$ & Fund I \\
\hline \multirow[t]{5}{*}{ Equity } & CHF & $0.0 \%$ & $0.0 \%$ & $0.0 \%$ & $20.0 \%$ & $5.0 \%$ & $3.0 \%$ & $27.0 \%$ & $7.0 \%$ & $5.0 \%$ \\
\hline & USD & $0.0 \%$ & $0.0 \%$ & $0.0 \%$ & $0.0 \%$ & $0.0 \%$ & $16.0 \%$ & $4.0 \%$ & $4.0 \%$ & $25.0 \%$ \\
\hline & EUR & $0.0 \%$ & $0.0 \%$ & $0.0 \%$ & $5.0 \%$ & $19.0 \%$ & $5.0 \%$ & $22.0 \%$ & $35.0 \%$ & $14.0 \%$ \\
\hline & JPY & $0.0 \%$ & $0.0 \%$ & $0.0 \%$ & $4.0 \%$ & $4.0 \%$ & $4.0 \%$ & $6.0 \%$ & $6.0 \%$ & $8.0 \%$ \\
\hline & GBP & $0.0 \%$ & $0.0 \%$ & $0.0 \%$ & $0.0 \%$ & $0.0 \%$ & $0.0 \%$ & $0.0 \%$ & $0.0 \%$ & $0.0 \%$ \\
\hline \multirow[t]{5}{*}{ Bonds } & CHF & $50.0 \%$ & $10.0 \%$ & $0.0 \%$ & $30.0 \%$ & $0.0 \%$ & $0.0 \%$ & $5.0 \%$ & $0.0 \%$ & $0.0 \%$ \\
\hline & USD & $15.0 \%$ & $15.0 \%$ & $70.0 \%$ & $0.0 \%$ & $0.0 \%$ & $43.0 \%$ & $0.0 \%$ & $0.0 \%$ & $29.0 \%$ \\
\hline & EUR & $35.0 \%$ & $75.0 \%$ & $20.0 \%$ & $20.0 \%$ & $54.0 \%$ & $14.0 \%$ & $10.0 \%$ & $32.0 \%$ & $5.0 \%$ \\
\hline & JPY & $0.0 \%$ & $0.0 \%$ & $0.0 \%$ & $4.0 \%$ & $0.0 \%$ & $0.0 \%$ & $0.0 \%$ & $0.0 \%$ & $0.0 \%$ \\
\hline & GBP & $0.0 \%$ & $0.0 \%$ & $0.0 \%$ & $0.0 \%$ & $0.0 \%$ & $0.0 \%$ & $12.0 \%$ & $0.0 \%$ & $0.0 \%$ \\
\hline \multirow[t]{8}{*}{ Liquidity } & $\mathrm{CHF}$ & $0.0 \%$ & $0.0 \%$ & $0.0 \%$ & $0.0 \%$ & $0.0 \%$ & $0.0 \%$ & $9.0 \%$ & $0.0 \%$ & $0.0 \%$ \\
\hline & USD & $0.0 \%$ & $0.0 \%$ & $10.0 \%$ & $17.0 \%$ & $13.0 \%$ & $12.0 \%$ & $5.0 \%$ & $5.0 \%$ & $10.0 \%$ \\
\hline & EUR & $0.0 \%$ & $0.0 \%$ & $0.0 \%$ & $0.0 \%$ & $4.0 \%$ & $4.0 \%$ & $0.0 \%$ & $11.0 \%$ & $4.0 \%$ \\
\hline & JPY & $0.0 \%$ & $0.0 \%$ & $0.0 \%$ & $0.0 \%$ & $0.0 \%$ & $0.0 \%$ & $0.0 \%$ & $0.0 \%$ & $0.0 \%$ \\
\hline & GBP & $0.0 \%$ & $0.0 \%$ & $0.0 \%$ & $0.0 \%$ & $0.0 \%$ & $0.0 \%$ & $0.0 \%$ & $0.0 \%$ & $0.0 \%$ \\
\hline & Other & $0.0 \%$ & $0.0 \%$ & $0.0 \%$ & $0.0 \%$ & $1.0 \%$ & $-1.0 \%$ & $0.0 \%$ & $0.0 \%$ & $0.0 \%$ \\
\hline & & $100.0 \%$ & $100.0 \%$ & $100.0 \%$ & $100.0 \%$ & $100.0 \%$ & $100.0 \%$ & $100.0 \%$ & $100.0 \%$ & $100.0 \%$ \\
\hline & & Fund J & Fund $\mathrm{K}$ & Fund $\mathrm{L}$ & Fund M & Fund N & Fund 0 & Fund $P$ & Fund Q & Fund R \\
\hline \multirow[t]{5}{*}{ Equity } & CHF & $40.0 \%$ & $10.0 \%$ & $8.0 \%$ & $0.0 \%$ & $0.0 \%$ & $0.0 \%$ & $0.0 \%$ & $0.0 \%$ & $0.0 \%$ \\
\hline & USD & $13.0 \%$ & $13.0 \%$ & $47.0 \%$ & $0.0 \%$ & $0.0 \%$ & $0.0 \%$ & $0.0 \%$ & $0.0 \%$ & $0.0 \%$ \\
\hline & EUR & $10.0 \%$ & $45.0 \%$ & $13.0 \%$ & $0.0 \%$ & $0.0 \%$ & $0.0 \%$ & $0.0 \%$ & $0.0 \%$ & $0.0 \%$ \\
\hline & JPY & $8.0 \%$ & $8.0 \%$ & $8.0 \%$ & $0.0 \%$ & $0.0 \%$ & $0.0 \%$ & $0.0 \%$ & $0.0 \%$ & $0.0 \%$ \\
\hline & GBP & $0.0 \%$ & $0.0 \%$ & $0.0 \%$ & $0.0 \%$ & $0.0 \%$ & $0.0 \%$ & $0.0 \%$ & $0.0 \%$ & $0.0 \%$ \\
\hline \multirow[t]{5}{*}{ Bonds } & $\mathrm{CHF}$ & $0.0 \%$ & $0.0 \%$ & $0.0 \%$ & $100.0 \%$ & $0.0 \%$ & $0.0 \%$ & $0.0 \%$ & $50.0 \%$ & $0.0 \%$ \\
\hline & USD & $4.0 \%$ & $0.0 \%$ & $8.0 \%$ & $0.0 \%$ & $100.0 \%$ & $0.0 \%$ & $50.0 \%$ & $50.0 \%$ & $50.0 \%$ \\
\hline & EUR & $9.0 \%$ & $9.0 \%$ & $0.0 \%$ & $0.0 \%$ & $0.0 \%$ & $100.0 \%$ & $50.0 \%$ & $0.0 \%$ & $10.0 \%$ \\
\hline & JPY & $0.0 \%$ & $0.0 \%$ & $0.0 \%$ & $0.0 \%$ & $0.0 \%$ & $0.0 \%$ & $0.0 \%$ & $0.0 \%$ & $40.0 \%$ \\
\hline & GBP & $0.0 \%$ & $0.0 \%$ & $0.0 \%$ & $0.0 \%$ & $0.0 \%$ & $0.0 \%$ & $0.0 \%$ & $0.0 \%$ & $0.0 \%$ \\
\hline \multirow[t]{8}{*}{ Liquidity } & $\mathrm{CHF}$ & $10.0 \%$ & $0.0 \%$ & $0.0 \%$ & $0.0 \%$ & $0.0 \%$ & $0.0 \%$ & $0.0 \%$ & $0.0 \%$ & $0.0 \%$ \\
\hline & USD & $5.0 \%$ & $5.0 \%$ & $11.0 \%$ & $0.0 \%$ & $0.0 \%$ & $0.0 \%$ & $0.0 \%$ & $0.0 \%$ & $0.0 \%$ \\
\hline & EUR & $0.0 \%$ & $10.0 \%$ & $5.0 \%$ & $0.0 \%$ & $0.0 \%$ & $0.0 \%$ & $0.0 \%$ & $0.0 \%$ & $0.0 \%$ \\
\hline & JPY & $0.0 \%$ & $0.0 \%$ & $0.0 \%$ & $0.0 \%$ & $0.0 \%$ & $0.0 \%$ & $0.0 \%$ & $0.0 \%$ & $0.0 \%$ \\
\hline & GBP & $0.0 \%$ & $0.0 \%$ & $0.0 \%$ & $0.0 \%$ & $0.0 \%$ & $0.0 \%$ & $0.0 \%$ & $0.0 \%$ & $0.0 \%$ \\
\hline & Other & $1.0 \%$ & $0.0 \%$ & $0.0 \%$ & $0.0 \%$ & $0.0 \%$ & $0.0 \%$ & $0.0 \%$ & $0.0 \%$ & $0.0 \%$ \\
\hline & & $100.0 \%$ & $100.0 \%$ & $100.0 \%$ & $100.0 \%$ & $100.0 \%$ & $100.0 \%$ & $100.0 \%$ & $100.0 \%$ & $100.0 \%$ \\
\hline & & Fund $\mathrm{S}$ & Fund T & Fund U & Fund V & Fund W & Fund $X$ & Fund $\mathbf{Y}$ & Fund $Z$ & \\
\hline \multirow[t]{5}{*}{ Equity } & CHF & $0.0 \%$ & $100.0 \%$ & $0.0 \%$ & $0.0 \%$ & $0.0 \%$ & $5.0 \%$ & $0.0 \%$ & $0.0 \%$ & \\
\hline & USD & $0.0 \%$ & $0.0 \%$ & $0.0 \%$ & $100.0 \%$ & $0.0 \%$ & $5.0 \%$ & $0.0 \%$ & $10.0 \%$ & \\
\hline & EUR & $0.0 \%$ & $0.0 \%$ & $100.0 \%$ & $0.0 \%$ & $0.0 \%$ & $60.0 \%$ & $30.0 \%$ & $0.0 \%$ & \\
\hline & JPY & $0.0 \%$ & $0.0 \%$ & $0.0 \%$ & $0.0 \%$ & $0.0 \%$ & $0.0 \%$ & $70.0 \%$ & $0.0 \%$ & \\
\hline & GBP & $0.0 \%$ & $0.0 \%$ & $0.0 \%$ & $0.0 \%$ & $0.0 \%$ & $30.0 \%$ & $0.0 \%$ & $0.0 \%$ & \\
\hline \multirow[t]{5}{*}{ Bonds } & $\mathrm{CHF}$ & $5.0 \%$ & $0.0 \%$ & $0.0 \%$ & $0.0 \%$ & $0.0 \%$ & $0.0 \%$ & $0.0 \%$ & $0.0 \%$ & \\
\hline & USD & $40.0 \%$ & $0.0 \%$ & $0.0 \%$ & $0.0 \%$ & $0.0 \%$ & $0.0 \%$ & $0.0 \%$ & $0.0 \%$ & \\
\hline & EUR & $25.0 \%$ & $0.0 \%$ & $0.0 \%$ & $0.0 \%$ & $0.0 \%$ & $0.0 \%$ & $0.0 \%$ & $0.0 \%$ & \\
\hline & JPY & $0.0 \%$ & $0.0 \%$ & $0.0 \%$ & $0.0 \%$ & $0.0 \%$ & $0.0 \%$ & $0.0 \%$ & $0.0 \%$ & \\
\hline & GBP & $30.0 \%$ & $0.0 \%$ & $0.0 \%$ & $0.0 \%$ & $0.0 \%$ & $0.0 \%$ & $0.0 \%$ & $0.0 \%$ & \\
\hline \multirow[t]{7}{*}{ Liquidity } & CHF & $0.0 \%$ & $0.0 \%$ & $0.0 \%$ & $0.0 \%$ & $0.0 \%$ & $0.0 \%$ & $0.0 \%$ & $0.0 \%$ & \\
\hline & USD & $0.0 \%$ & $0.0 \%$ & $0.0 \%$ & $0.0 \%$ & $0.0 \%$ & $0.0 \%$ & $0.0 \%$ & $0.0 \%$ & \\
\hline & EUR & $0.0 \%$ & $0.0 \%$ & $0.0 \%$ & $0.0 \%$ & $0.0 \%$ & $0.0 \%$ & $0.0 \%$ & $0.0 \%$ & \\
\hline & JPY & $0.0 \%$ & $0.0 \%$ & $0.0 \%$ & $0.0 \%$ & $0.0 \%$ & $0.0 \%$ & $0.0 \%$ & $0.0 \%$ & \\
\hline & GBP & $0.0 \%$ & $0.0 \%$ & $0.0 \%$ & $0.0 \%$ & $0.0 \%$ & $0.0 \%$ & $0.0 \%$ & $0.0 \%$ & \\
\hline & Other & $0.0 \%$ & $0.0 \%$ & $0.0 \%$ & $0.0 \%$ & $100.0 \%$ & $0.0 \%$ & $0.0 \%$ & $90.0 \%$ & \\
\hline & & $100.0 \%$ & $100.0 \%$ & $100.0 \%$ & $100.0 \%$ & $100.0 \%$ & $100.0 \%$ & $100.0 \%$ & $100.0 \%$ & \\
\hline
\end{tabular}

TABLE 5. The asset allocation of the various funds. All numbers in percentage of wealth (converted in "home currency" where necessary). 